Introduction Respiratory protective devices are used to protect workers from inhalation of hazardous atmospheres. Beside the aspects related to adequacy of the device in protecting the worker, it is necessary to investigate also the aspects related to the suitability of the device such as ergonomic factors: one of the most common complain of the workers is related to the face thermal sensation perceived as hot.

In this study, the effect of a power assisted filtering device incorporating a full face mask on the thermophysiological response of a thermal manikin is investigated.

Methods The experimental protocol schedules tests on a standing sweating thermal manikin (Newton) in the climatic chamber, where hot conditions were simulated (air temperature at $34^{\circ} \mathrm{C}$, relative humidity at $\left.32 \%\right)$. Two conditions were tested: manikin with the respirator powered on (CR) and manikin without the respirator (CC). Two different levels of metabolic rate were set (1 MET and 3 METs).

Results Results show:

- at 1 MET the manikin is in thermal balance with the external environment with or without respirator.

- at $3 \mathrm{MET}$, although the thermoregulation system is very stressed (the rectal temperature increases continuously with or without respirator), the effect of the respirator produces a small decrease of the local and global temperatures and also of the amount of sweat generated by active thermoregulation that reaches its maximum value of $30 \mathrm{~g} / \mathrm{min}$ in $\mathrm{CC}$ ). The body reaches sensation between 'hot' (CC) and 'warm' (CR) while the face sensation increases rapidly reaching the value correspondent to 'very hot' both for CC and CR.

Discussion At $M=1$ MET and $M=3$ MET the effect of the hot environment is weakened by the action of the respirator which allows a continuous heat exchange removing and renovating the air layer around the face without providing an additional thermal load.

\section{SEASONAL CHANGES OF PHYSIOLOGICAL RESPONSES ASSOCIATED WITH HEAT ACCLIMATISATION}

${ }^{1}$ Shota Tabuchi*, ${ }^{2}$ Shoko Kawanami, 'Daisuke Inoue, 'Shuhei Morizane, ${ }^{1}$ Jinro Inoue, ${ }^{1}$ Seichi Horie. 'Department of Health Policy and Management, University of Occupational and Environmental Health, Japan; ${ }^{2}$ Occupational Health Training Centre, University of Occupational and Environmental Health, Japan

\subsection{6/oemed-2018-ICOHabstracts. 1428}

Introduction It is known that heat stroke is likely to occur when workers are not acclimatised to heat. The purpose of this study is to clarify if the physiological responses of heat acclimatisation are different by the seasonal difference of the temperature in living environment.

Methods Four healthy males in twenties repeated a 20 minutes-exercise on an ergometer at $40 \% \mathrm{VO}_{2} \mathrm{max}$ for three times in three different thermal environments in the artificial climate room controlled at $28^{\circ} \mathrm{C}$, then elevated to $34^{\circ} \mathrm{C}$ and finally to $40^{\circ} \mathrm{C}$ where relative humidity was fixed at $50 \%$. Each exercise was followed by 20 minutes-rest period kept seated in the adjunct room at $24^{\circ} \mathrm{C}$. They were asked to exercise consecutively for five days. This series of intervention was repeated during summer and winter seasons. We continuously measured their auditory canal temperature $\left(t_{\mathrm{ac}}\right)$ and heart rate (HR). We also examined their sweat $\mathrm{Na}^{+}$and $\mathrm{K}^{+}$concentration and estimated their sweat volume from the body weight loss.
Result The observed $t_{\mathrm{ac}}$ and HR at the end of the exercise were generally higher in winter compared to summer. The sweat volume gradually increased for five days and the volume during the first exercise period at $28^{\circ} \mathrm{C}$ was larger in summer; however, the amount of increase was larger in winter. The sweat $\mathrm{Na}^{+}$concentration positively correlated with sweat volume and we observed smaller elevation of $\mathrm{Na}^{+}$concentration along with the increase of sweat volume in summer.

Discussion The lower $t_{\mathrm{ac}}$ and $\mathrm{HR}$, earlier sweating, and smaller elevation of $\mathrm{Na}^{+}$concentration during heat acclimatisation in summer seem to be caused by the different thermal condition of living environment

\section{PEOPLE AT ELEVATED RISK OF DEVELOPING HEAT- RELATED ILLNESS AT WORKPLACE: A CASE-CONTROL STUDY}

${ }^{1} S$ Horie*, ${ }^{2} \mathrm{~N}$ Gommori, 'S Tabuchi, 'J Inoue, 'S Kawanami. 'University of Occupational and Environmental Health, Kitakyushu, Japan; ${ }^{2}$ JfE Steel Corporation, Kawasaki, Japan

10.1136/oemed-2018-ICOHabstracts. 1429

Introduction Primary causes of heat-related illness (HRI) at work are:

- extreme heat and humidity,

- heavy work load,

- continuous exertion and infrequent rest,

- clothes with poor vapor-permeability, and

- personal health condition.

Empirical research has not been performed to investigate the effect of underlying health problems to HRI.

Methods A case-control study was performed by asking occupational health staffs to report all HRI cases occurred at their workplaces in 2015 and 2016. One co-worker control of the same gender at the closest in age was also reported for each case. Target population included:

- 26 large manufacturing factories,

- 15 major construction companies,

- all traffic guard companies in Japan, and

- a fire stations in Kitakyushu city.

Their results of the periodic health examination were collected to evaluate the underlying health problems of obesity (BMI >25), impaired glucose tolerance, IGT (HbA1c $\geq 6.5 \%$, fast blood sugar $\geq 126 \mathrm{mg} / \mathrm{dL}$, and/or casual blood sugar $\geq 200 \mathrm{mg} / \mathrm{dL})$, high blood pressure, HBP $(\geq 140 / 90 \mathrm{mmHg})$, and dyslipidemia (LDL-C $\geq 140 \mathrm{mg} / \mathrm{dL}$, HDL-C $<40 \mathrm{mg} / \mathrm{dL}$, or $\mathrm{TG} \geq 150 \mathrm{mg} / \mathrm{dL}$ ). Data were statistically compared between the case and the control using JMP Pro 13.

Results Overall incidence rate of HRI at the examined workplaces was $0.065 \%$ (114 cases/1 76094 person-year). A total of 102 pairs $(n=204)$ could be compared. Cases showed higher BMI $(p=0.046)$ and visceral circumference $(p=0.045)$. Cases and controls included 12 and 2 persons with IGT, respectively $(p=0.005)$. Multiple logistic regression analysis revealed increase of $\mathrm{HbA} 1 \mathrm{c}$ elevated the risk of developing HRI (OR 16.76 [95\% CI: 2.44 to 165.5], $p=0.0014$ ), whereas LDL-cholesterol showed protective effect (OR 0.97 [0.95-0.99], $\mathrm{p}<0.001)$.

Conclusion Significantly elevated risk of developing heatrelated illness was observed among workers with IGT. 